\title{
Elements of order 4 of the Hilbert kernel in quadratic number fields
}

\author{
by \\ QIN YuE (Shanghai and Jiangsu)
}

1. Introduction. Let $O_{F}$ be the ring of integers of a number field $F$. Let $A$ be a finite Abelian group. We denote the 2-Sylow subgroup of $A$ by $A_{2}$, the 2-rank of $A$ by $r_{2}(A)$, and the 4-rank of $A$ by $r_{4}(A)$.

By $[2,5,9]$, we have 2-rank and 4-rank formulas for $K_{2} O_{F}$. For quadratic fields, Browkin and Schinzel [3] have given 2-rank formulas and forms of elements of order 2 of $K_{2} O_{F}$; Qin $[12,13,14]$ has obtained a method to calculate 4-ranks of $\mathrm{K}_{2} \mathrm{O}_{F}$. Recently, Hurrelbrink and Kolster [8] have presented an effective way of computing 4-ranks of $\mathrm{K}_{2} \mathrm{O}_{F}$ for relative quadratic extensions via the determination of the $F_{2}$-ranks of certain matrices of local Hilbert symbols. In [17] we have proved the following formula:

$$
r_{4}\left(K_{2} O_{F}\right)=a(F)+r_{4}(C(E)),
$$

where $F=\mathbb{Q}(\sqrt{d}), E=\mathbb{Q}(\sqrt{-d}), a(F)=-1,0$, or 1 is a constant determined effectively by the Rédei matrices of $E$, and $C(E)$ is the narrow class group of $E$.

In the present paper, we concentrate on the structure of the 2-Sylow subgroup of $\mathrm{K}_{2} \mathrm{O}_{F}$ and use the method of $[5,9]$ to give the results of $[12,13$, $14]$ and to express the forms of elements of order 4 of $K_{2} O_{F}$ for quadratic fields $F$, which are simpler. Using these forms we discuss whether elements of order 4 of $K_{2} O_{F}$ are contained in Hilbert kernel $\Re_{2} F$. Hence, we get the relation between $r_{4}\left(K_{2} O_{F}\right)$ and $r_{4}\left(\Re_{2} F\right)$ and we get some quadratic fields with elements of order 8 in $K_{2} O_{F}$. We also obtain the following result: if $F=\mathbb{Q}\left(\sqrt{p_{1} p_{2}}\right)$, where $p_{1}$ and $p_{2}$ are primes with $p_{1} \neq p_{2}, p_{1} \equiv p_{2} \equiv 5 \bmod 8$, then $K_{2} O_{F} \cong \mathbb{Z} /(2) \oplus \mathbb{Z} /(2) \oplus \mathbb{Z} /(4)$ if and only if $16 \mid h\left(-p_{1} p_{2}\right)$, where $h\left(-p_{1} p_{2}\right)$ is the class number of $E=\mathbb{Q}\left(\sqrt{-p_{1} p_{2}}\right)$. For imaginary quadratic fields, we add some values of the Tate kernel to the tables of [13].

2000 Mathematics Subject Classification: 11R65, 11R70, 19C99, 19D50.

The paper is supported by Morningside Center of Math., CAS, Shanghai Postdoctoral Science Foundation, and 00KJB110006. 
2. Elements of order 4 in the tame kernel. We use the method of $[5,9]$ to investigate the elements of order 4 of $K_{2} O_{F}$ for quadratic fields $F$. Now, we describe the notations of [5]:

- $F=\mathbb{Q}(\sqrt{d}), E=\mathbb{Q}(\sqrt{-d}), M=F(i), d>2$ a squarefree integer.

- $S$ is the set of infinite and dyadic places of $F$.

- $G_{F}=\left\{\operatorname{cl}(b) \in F^{*} / F^{* 2} \mid v_{P}(b) \equiv 0 \bmod 2\right.$ for all $\left.P \notin S\right\}$.

- $H_{F}=\left\{\operatorname{cl}(b) \in G_{F} \mid b \in N_{M / F}\left(M^{*}\right)\right\}$.

In [5], there are defined maps:

$$
\begin{gathered}
\chi_{1}, \chi_{2}: H_{F} \rightarrow C_{S}(F) / C_{S}^{2}(F), \\
\chi_{1}: \operatorname{cl}(b) \mapsto\left[\prod_{P \notin S} P^{v_{P}(b) / 2}\right], \quad \chi_{2}: \operatorname{cl}(b) \mapsto\left[\prod_{P \notin S} P^{v_{\mathcal{P}}(\alpha)}\right],
\end{gathered}
$$

where $C_{S}(F)$ is the $S$-ideal class group of $F, N_{M / F}(\alpha)=b$ for $\alpha \in M$, and $\mathcal{P}$ is a place of $M$ over $P$. Let $\chi=\chi_{1} \chi_{2}$. Then ker $\chi$ is determined by the elements of order 4 of $K_{2} O_{F}$ and the elements $a \in F^{*}$ with $\{-1, a\}=1$ (see [5], Prop. 2.3, or [9], Prop. 1.5).

Browkin-Schinzel ([3], Theorem 2) gave the elements of order at most 2 of $\mathrm{K}_{2} \mathrm{O}_{F}$ for a real quadratic field $F=\mathbb{Q}(\sqrt{d})$ :

$$
\left\{-1, m \gamma_{j}\right\} \text {, }
$$

where $m$ is an odd divisor of $d$ and $\gamma_{j}=u_{j}+\sqrt{d}$ with $u_{j}^{2}-j w_{j}^{2}=d$, $u_{j}, w_{j} \in \mathbb{N}, j \in N_{F / \mathbb{Q}}\left(F^{*}\right) \cap\{-1, \pm 2\}, \gamma_{1}=1$. We denote $N_{F / \mathbb{Q}}\left(F^{*}\right)$ by $N F$.

By Bass-Tate theorem [10], $\beta \in K_{2} F, \beta^{2}=\left\{-1, m \gamma_{j}\right\}$ if and only if $m \gamma_{j} \in N_{M / F}\left(M^{*}\right)$. On the other hand, for all $P \notin S$, the tame symbols $\tau_{P}\left\{-1, m \gamma_{j}\right\}$ equal 1 , so the Hilbert symbols $\eta_{P}\left(\left\{-1, m \gamma_{j}\right\}\right)$ are 1 by [2], Theorem 2. By the Minkowski-Hasse theorem, we know that: if $d \not \equiv 1 \bmod 8$, then $m \gamma_{j} \in N_{M / F}\left(M^{*}\right)$ if and only if $m>0$ and $j=1,2$; if $d \equiv 1 \bmod 8$ and $2 \notin N F$, then $m \gamma_{j} \in N_{M / F}\left(M^{*}\right)$ if and only if $m>0$ and $j=1$; if $d \equiv 1 \bmod 8$ and $u^{2}-2 w^{2}=d$, where $u, w \in \mathbb{N}, w \equiv 0 \bmod 4$, then $m \gamma_{j} \in N_{M / F}\left(M^{*}\right)$ if and only if either $j=1, m>0$, and $m \equiv 1 \bmod 4$, or $j=2, m>0$, and $m+u \equiv 2 \bmod 4$.

Suppose that $\beta \in K_{2} F$ and

$$
\beta^{2}=\left\{-1, m \gamma_{j}\right\} \in K_{2} O_{F} .
$$

We will find conditions sufficient for $\beta \in K_{2} \mathrm{O}_{2}$.

CASE 1: $j=1$ and $m$ is an odd positive divisor of $d$ in (2.1). Since $m \in$ $N_{M / F}\left(M^{*}\right)$, there are $X=x_{1}+x_{2} \sqrt{d}, Y=y_{1}+y_{2} \sqrt{d} \in F$ and $x_{1}, x_{2}, y_{1}, y_{2}$ $\in \mathbb{Q}$ such that

$$
m=X^{2}+Y^{2}=\left(x_{1}^{2}+y_{1}^{2}\right)+\left(x_{2}^{2}+y_{2}^{2}\right) d+2\left(x_{1} x_{2}+y_{1} y_{2}\right) \sqrt{d} .
$$


Hence $x_{1} x_{2}+y_{1} y_{2}=0$. First we assume that $x_{1}, x_{2}, y_{1}, y_{2}$ are all non-zero, and put $t=x_{1} / y_{1}=-y_{2} / x_{2}$. By the last equality, $m=\left(1+t^{2}\right)\left(y_{1}^{2}+x_{2}^{2} d\right)$. Therefore, there is a squarefree positive integer $k$, with each odd prime factor $p_{i} \equiv 1 \bmod 4$, such that the Diophantine equation

$$
m k z^{2}=x^{2}+d y^{2}
$$

is solvable in $\mathbb{Z}$. If $x_{1}=y_{1}=0$, take $k=d / m$; if $x_{2}=y_{2}=0$, take $k=m$; if $x_{1}=y_{2}=0$ or $x_{2}=y_{1}=0$, take $k=1$.

When $k \geq 2$, there are $g, h \in \mathbb{N}$ such that

$$
k=g^{2}+h^{2} \text {. }
$$

Take a relatively prime solution $(x, y, z)=(a, b, c)$ of the equation $(2.2)$ in $\mathbb{N}$. Put $\alpha_{1}=a+b \sqrt{-d}, \alpha_{2}=g+h i$, and $\alpha=\alpha_{1} \alpha_{2}$. Then $N_{M / F}(\alpha)=m k^{2} c^{2}$ and $\operatorname{cl}(m)=\operatorname{cl}\left(m k^{2} c^{2}\right) \in H_{F}$. Below, we discuss the value of $\chi(\operatorname{cl}(m))$. For convenience, let $p$ be an odd prime, $P$ a place of $F$ over $p$, and $\mathcal{P}$ a place of $M$ over $P$, which we denote by $P \mid p$ and $\mathcal{P} \mid P$. Suppose $p \mid m k^{2} c^{2}$.

(i) If $p \nmid k, p \mid m$, then $p \mid a, p \nmid b, p \nmid c$ for the relatively prime solution $(x, y, z)=(a, b, c)$ of $(2.2)$ in $\mathbb{N}$. Hence $v_{P}\left(m k^{2} c^{2}\right) / 2=v_{P}(m) / 2=1$ and $v_{\mathcal{P}}(\alpha)=v_{\mathcal{P}}\left(\alpha_{1}\right)+v_{\mathcal{P}}\left(\alpha_{2}\right)=1+0=1$.

(ii) If $p \nmid k, p \mid c$, then $p \nmid d, p \nmid a, p \nmid b$. Hence $v_{P}\left(m k^{2} c^{2}\right) / 2=v_{p}(c)$ and $v_{\mathcal{P}}(\alpha) \equiv 0+0=0 \bmod 2$.

(iii) If $p|k, p| m$, then $p \| a, p \mid b, p \nmid c$. Hence $v_{P}\left(m k^{2} c^{2}\right) / 2 \equiv 1 \bmod 2$ and $v_{\mathcal{P}}(\alpha) \equiv 0+0 \equiv 0 \bmod 2$.

(iv) If $p|k, p \nmid m, p| d$, then $p \mid a, p \nmid b, p \nmid c$. Hence $v_{P}\left(m k^{2} c^{2}\right) / 2=$ $v_{P}(k) \equiv 0 \bmod 2$ and $v_{\mathcal{P}}(\alpha) \equiv 1+0 \equiv 1 \bmod 2$.

(v) If $p \mid k, p \nmid d$, then $p \nmid a, p \nmid b$ in both cases $p \mid c$ and $p \nmid c$. Hence $v_{P}\left(m k^{2} c^{2}\right) / 2=v_{P}(k)+v_{P}(c) \equiv 1+v_{p}(c) \bmod 2$. In this case, we investigate the value of $v_{\mathcal{P}}(\alpha)$.

There is a diagram of field extensions

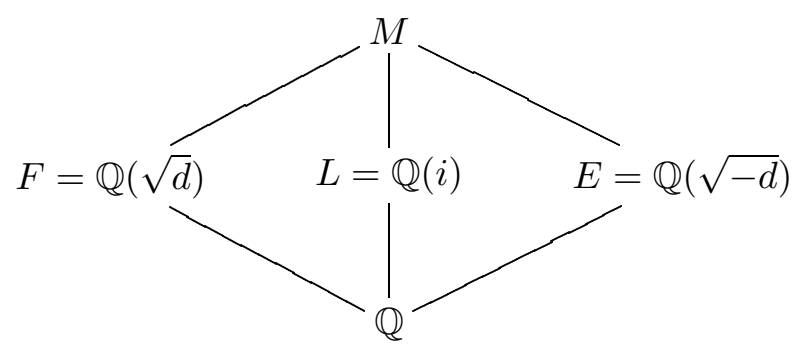

Since $p$ splits in $E$ and $L, p$ splits completely in $M$. Let $\operatorname{Gal}(M / \mathbb{Q})=$ $\{1, \sigma, \varrho, \sigma \varrho\}$ be the Galois group of the finite extension $M / \mathbb{Q}$, where $\sigma$ : $\sqrt{d} \mapsto \sqrt{d}, i \mapsto-i$ and $\varrho: \sqrt{d} \mapsto-\sqrt{d}, i \mapsto-i$. Then $p O_{M}=\mathcal{P}_{1} \mathcal{P}_{2} \mathcal{P}_{3} \mathcal{P}_{4}$, $\mathcal{P}_{2}=\sigma \mathcal{P}_{1}, \mathcal{P}_{3}=\varrho \mathcal{P}_{1}, \mathcal{P}_{4}=\sigma \varrho \mathcal{P}_{1}, p O_{F}=P_{1} P_{2}, P_{1} O_{M}=\mathcal{P}_{1} \mathcal{P}_{2}, P_{2} O_{M}=$ 
$\mathcal{P}_{3} \mathcal{P}_{4}$. Hence we have, modulo 2 ,

$$
\begin{aligned}
& \left\{\begin{array} { l } 
{ v _ { \mathcal { P } _ { 1 } } ( \alpha _ { 1 } ) = v _ { \mathcal { P } _ { 3 } } ( \alpha _ { 1 } ) \equiv 0 , } \\
{ v _ { \mathcal { P } _ { 2 } } ( \alpha _ { 1 } ) = v _ { \mathcal { P } _ { 4 } } ( \alpha _ { 1 } ) \equiv 1 , }
\end{array} \quad \text { or } \quad \left\{\begin{array}{l}
v_{\mathcal{P}_{1}}\left(\alpha_{1}\right)=v_{\mathcal{P}_{3}}\left(\alpha_{1}\right) \equiv 1, \\
v_{\mathcal{P}_{2}}\left(\alpha_{1}\right)=v_{\mathcal{P}_{4}}\left(\alpha_{1}\right) \equiv 0,
\end{array}\right.\right. \\
& \left\{\begin{array} { l } 
{ v _ { \mathcal { P } _ { 1 } } ( \alpha _ { 2 } ) = v _ { \mathcal { P } _ { 4 } } ( \alpha _ { 2 } ) \equiv 0 , } \\
{ v _ { \mathcal { P } _ { 2 } } ( \alpha _ { 2 } ) = v _ { \mathcal { P } _ { 3 } } ( \alpha _ { 2 } ) \equiv 1 , }
\end{array} \quad \text { or } \quad \left\{\begin{array}{l}
v_{\mathcal{P}_{1}}\left(\alpha_{2}\right)=v_{\mathcal{P}_{4}}\left(\alpha_{2}\right) \equiv 1 \\
v_{\mathcal{P}_{2}}\left(\alpha_{2}\right)=v_{\mathcal{P}_{3}}\left(\alpha_{2}\right) \equiv 0 .
\end{array}\right.\right.
\end{aligned}
$$

Therefore

$$
\left\{\begin{array} { l } 
{ v _ { \mathcal { P } _ { 1 } } ( \alpha ) = v _ { \mathcal { P } _ { 2 } } ( \alpha ) \equiv 0 , } \\
{ v _ { \mathcal { P } _ { 3 } } ( \alpha ) = v _ { \mathcal { P } _ { 4 } } ( \alpha ) \equiv 1 , }
\end{array} \quad \text { or } \quad \left\{\begin{array}{l}
v_{\mathcal{P}_{1}}(\alpha)=v_{\mathcal{P}_{2}}(\alpha) \equiv 1 \\
v_{\mathcal{P}_{3}}(\alpha)=v_{\mathcal{P}_{4}}(\alpha) \equiv 0
\end{array}\right.\right.
$$

Consequently, $\chi(\operatorname{cl}(m))=[c I]=[I]$, where $I \bar{I}=k O_{F}, \bar{I}$ a conjugate ideal of $I$. Hence $\operatorname{cl}(m) \in \operatorname{ker} \chi$ if and only if $[I] \in C_{S}^{2}(F)$. Let $H(F)$ be the narrow class group of $F$. Then, by the Gauss theorem, $[J] \in H^{2}(F)$, where $J$ is an ideal of $F$, if and only if $N_{F / \mathbb{Q}}(J) \in N F$. On the other hand, let $[A]$ be the narrow class containing the ideal $A=(\sqrt{d})$ and $[B]$ the narrow class containing $B \mid 2$. Put $H_{2}(F)=\langle[A],[B]\rangle$, the group generated by $[A],[B]$. Then

$$
C_{S}(F)=H(F) / H_{2}(F) \text {. }
$$

Therefore, we have

$$
\begin{aligned}
\operatorname{cl}(m)=\operatorname{cl} & \left(m k^{2} c^{2}\right) \in \operatorname{ker} \chi \\
& \Leftrightarrow[I] \in C_{S}^{2}(F), \text { i.e., }[I][X] \in H^{2}(F), \text { where }[X] \in H_{2}(F) \\
& \Leftrightarrow N_{F / \mathbb{Q}}(I X) \in N F, \text { i.e., } k \varepsilon \in N F, \text { where } \varepsilon \in\{ \pm 1, \pm 2\} \\
& \Leftrightarrow \text { the following equation is solvable in } \mathbb{Z}:
\end{aligned}
$$

$$
\varepsilon k z^{2}=x^{2}-d y^{2} .
$$

By (2.2) and (2.5), we get:

Theorem 2.1 ([14], Theorem 2.2). Let $F=\mathbb{Q}(\sqrt{d}), d>2$ a squarefree integer. Then, for every odd positive divisor $m$ of $d$, there is $\beta \in K_{2} O_{F}$ with $\beta^{2}=\{-1, m\}$ if and only if there is $\varepsilon \in\{ \pm 1, \pm 2\}$ such that

$$
\left(\frac{\varepsilon d m^{-1}}{p}\right)=\left(\frac{\varepsilon m}{l}\right)=1 \quad \text { for any odd primes } p|m, l| d m^{-1} \text {. }
$$

By [9], Prop. 1.5, and the preceding argument, we can find $y \in F^{*}$ such that $v_{P}\left(N_{M / F}(\alpha)\right) / 2+v_{\mathcal{P}}(\alpha)+v_{P}(y) \equiv 0 \bmod 2$ for all $P \notin S$. Set

$$
\begin{aligned}
\beta & =\operatorname{tr}_{M / F}(\{i, \alpha\})\{-1, y\} \\
& =\operatorname{tr}_{M / F}\left(\left\{i, \alpha_{1}\right\}\right) \operatorname{tr}_{M / F}\left(\left\{i, \alpha_{2}\right\}\right)\{-1, y\} \\
& =\left\{-\frac{\sqrt{d} b}{a}, \frac{k m c^{2}}{a^{2}}\right\}\left\{-\frac{h}{g}, \frac{k}{g^{2}}\right\}\{-1, c \delta\}\{-1, e+f \sqrt{d}\},
\end{aligned}
$$


where $(x, y, z)=(e, f, t)$ is a relatively prime solution of $(2.5)$ in $\mathbb{N}$ and $\delta \mid k$, $\delta \in \mathbb{N}$. Then $\beta \in K_{2} O_{F}$ and $\beta^{2}=\{-1, m\}$.

In particular, suppose that $(m, \varepsilon), \varepsilon>0$, satisfies (2.6). Then take $k=\varepsilon$ in $(2.2)$ and set

$$
\beta=\left\{\frac{-\sqrt{d} b}{a}, \frac{\varepsilon m c^{2}}{a^{2}}\right\}\{-1, c\},
$$

where $(x, y, z)=(a, b, c)$ is a relatively prime solution of $\varepsilon m z^{2}=x^{2}+d y^{2}$ in $\mathbb{N}$. Then $\beta \in K_{2} O_{F}$ and $\beta^{2}=\{-1, m\}$.

CASE 2: $j=2$ and $m$ is an odd positive divisor of $d$ in (2.1). Since $2 \in N F$, we have $u^{2}-2 w^{2}=d, u, w \in \mathbb{N}$, and $\gamma_{2}=u+\sqrt{d}$. If $d \equiv 1 \bmod 8$, we take $w \equiv 0 \bmod 4$. Hence,

$$
\begin{aligned}
& w^{2}+(u+w+\sqrt{d})^{2}=2(u+w)(u+\sqrt{d}), \\
& (u+2 w)^{2}+d=2(u+w)^{2}
\end{aligned}
$$

so $d$ and $u+w$ are relatively prime. We assume $m \gamma_{2} \in N_{M / F}\left(M^{*}\right)$. Then $(u+w) m \in N_{M / F}\left(M^{*}\right)$ by (2.9). By the same method as in the first case, there is a squarefree positive integer $k$, with each odd prime divisor $p_{i} \equiv$ $1 \bmod 4$, such that the Diophantine equation

$$
m(u+w) k z^{2}=x^{2}+d y^{2}, \quad k=g^{2}+h^{2}, g, h \in \mathbb{N},
$$

is solvable in $\mathbb{Z}$. Take $\alpha_{1}=w+(u+w+\sqrt{d}) i, \alpha_{2}=a+b \sqrt{-d}, \alpha_{3}=g+h i$, $\alpha=\alpha_{1} \alpha_{2} \alpha_{3}$, where $(x, y, z)=(a, b, c)$ is a relatively prime solution of $(2.10)$ in $\mathbb{N}$. Then $N_{M / F}(\alpha)=2 m(u+\sqrt{d}) k^{2}(u+w)^{2} c^{2}$ and $\operatorname{cl}(m(u+\sqrt{d}))=$ $\operatorname{cl}\left(N_{M / F}(\alpha)\right) \in H_{F}$. We discuss the value of $\chi(\operatorname{cl}(m(u+\sqrt{d})))$. For $p$ an odd prime, let $P \mid p$ in $F$ and $\mathcal{P} \mid P$ in $M$. Suppose $P \mid N_{M / F}(\alpha)$. There are the following cases:

(i) If $p \nmid k, p \mid m$, then $p \nmid u+w, p \mid a, p \nmid b, p \nmid c, P \nmid u+\sqrt{d}$ for the relatively prime solution $(x, y, z)=(a, b, c)$ of $(2.10)$ in $\mathbb{N}$. Hence $v_{P}\left(N_{M / F}(\alpha)\right) / 2=$ $v_{P}(m) / 2=1$ and $v_{\mathcal{P}}(\alpha)=v_{\mathcal{P}}\left(\alpha_{2}\right)=v_{\mathcal{P}}(a+b \sqrt{-d})=1$.

(ii) If $p \nmid k, P \mid u+\sqrt{d}$, then $p \nmid u+w, p \nmid d$. Hence $v_{P}\left(N_{M / F}(\alpha)\right) / 2=$ $v_{P}(u+\sqrt{d}) / 2+v_{p}(c)$ and

$$
\begin{aligned}
v_{\mathcal{P}}(\alpha) & \equiv v_{\mathcal{P}}\left(\alpha_{1}\right)+0 \equiv v_{\mathcal{P}}((u+\sqrt{d}) i+w(1+i)) \equiv v_{\mathcal{P}}(w) \\
& \equiv v_{P}(u+\sqrt{d}) / 2 \bmod 2
\end{aligned}
$$

by $(2.9)$ and $(u+\sqrt{d})(u-\sqrt{d})=2 w^{2}$.

(iii) If $p \nmid k, p \mid u+w$, then $P \nmid u+\sqrt{d}, p \nmid d$. Without loss of generality, assume $p \nmid a, p \nmid b$. Hence $v_{P}\left(N_{M / F}(\alpha)\right) / 2=v_{p}(u+w)+v_{p}(c)$ and

$$
\begin{aligned}
v_{\mathcal{P}}(\alpha) & =v_{\mathcal{P}}\left(\alpha_{1}\right)+v_{\mathcal{P}}\left(\alpha_{2}\right)=v_{\mathcal{P}}((u+w) i+(w+\sqrt{-d}))+v_{\mathcal{P}}\left(\alpha_{2}\right) \\
& =v_{\mathcal{P}}((w+\sqrt{-d})(a+b \sqrt{-d})) .
\end{aligned}
$$


(iv) If $p|k, p| m$, then $p \| a, p \mid b, p \nmid c$. Hence $v_{P}\left(N_{M / F}(\alpha)\right) / 2=v_{P}(m) / 2$ $+v_{P}(k) \equiv 1 \bmod 2$ and $v_{\mathcal{P}}(\alpha)=v_{\mathcal{P}}\left(\alpha_{2}\right)+v_{\mathcal{P}}\left(\alpha_{3}\right) \equiv 0+0=0 \bmod 2$.

(v) If $p|k, p| d, p \nmid m$, then $p|a, p \nmid b, p| c$. Hence $v_{P}\left(N_{M / F}(\alpha)\right) / 2=$ $v_{P}(k) \equiv 0 \bmod 2$ and $v_{\mathcal{P}}(\alpha)=v_{\mathcal{P}}\left(\alpha_{2}\right)+v_{\mathcal{P}}\left(\alpha_{3}\right) \equiv 1+0=1 \bmod 2$.

(vi) If $p \mid k, p \nmid d$, then $p \nmid a, p \nmid b$. Hence we have $v_{P}\left(N_{M / F}(\alpha)\right) / 2=$ $v_{p}(k)+v_{p}(u+w)+v_{P}(u+\sqrt{d}) / 2+v_{p}(c)$. Suppose $P \mid u+\sqrt{d}$. Then $v_{\mathcal{P}}\left(\alpha_{1}\right)=$ $v_{\mathcal{P}}(u+\sqrt{d}+w(1+i))=v_{p}(w)=v_{P}(u+\sqrt{d}) / 2$ as $(u+\sqrt{d})(u-\sqrt{d})=2 w^{2}$. By the process of proving $(\mathrm{v})$ in the first case, we can get the same result for $v_{\mathcal{P}}\left(\alpha_{2} \alpha_{3}\right)$ as in (2.4). Suppose $p \mid u+w$ and, without loss of generality, assume $p \nmid a, p \nmid b$. Then $v_{\mathcal{P}}\left(\alpha_{1} \alpha_{2}\right)=v_{\mathcal{P}}((w+\sqrt{-d})(a+b \sqrt{-d}))$ by (iii). By the process of proving $(\mathrm{v})$ in Case 1 , we can get the same result for $v_{\mathcal{P}}(\alpha)$ as in (2.4). Suppose $p \nmid u+w, P \nmid u+\sqrt{d}$. Then we can get the same result for $v_{\mathcal{P}}(\alpha)$ as in $(2.4)$.

Consequently, $\chi(\operatorname{cl}(m(u+\sqrt{d})))=\left[c \delta_{1} I\right]$, where $\delta_{1} \mid u+w$ from (iii) and $I \bar{I}=k O_{F}, \bar{I}$ a conjugate ideal of $I$. By the method of Case 1 , we have $\chi(\operatorname{cl}(m(u+\sqrt{d}))) \in \operatorname{ker} \chi$ if and only if the following equation is solvable in $\mathbb{Z}, \varepsilon \in\{ \pm 1\}$ :

$$
\varepsilon k z^{2}=x^{2}-d y^{2}
$$

By (2.10) and (2.11), we get

TheOREM 2.2 ([14], Theorem 3.3). Let $F=\mathbb{Q}(\sqrt{d}), d>2$ a squarefree integer. Suppose that $d=u^{2}-2 w^{2}$ with $u, w \in \mathbb{N}$. Then, for every odd positive divisor $m$ of $d$, there is $\beta \in K_{2} O_{F}$ with $\beta^{2}=\{-1, m(u+\sqrt{d})\}$ if and only if there is $\varepsilon \in\{ \pm 1\}$ such that

$$
\begin{array}{ll}
\left(\frac{\varepsilon d m^{-1}(u+w)}{p}\right)=1 & \text { for every odd prime } p \mid m, \\
\left(\frac{\varepsilon m(u+w)}{l}\right)=1 & \text { for every odd prime } l \mid d m^{-1} .
\end{array}
$$

Suppose that $(m, \varepsilon)$ satisfies (2.12). Then, by [9], Prop. 1.5 and the preceding argument, we can find $y \in F^{*}$ such that $v_{P}\left(N_{M / F}(\alpha)\right) / 2+v_{\mathcal{P}}(\alpha)+$ $v_{P}(y) \equiv 0 \bmod 2$ for all $P \notin S$. Set

$$
\begin{aligned}
\beta= & \left\{-\frac{u+w+\sqrt{d}}{w}, \frac{2(u+w)(u+\sqrt{d})}{w^{2}}\right\} \\
& \times\left\{-\frac{b \sqrt{d}}{a}, \frac{m(u+w) k c^{2}}{a^{2}}\right\} \\
& \times\left\{-\frac{h}{g}, \frac{k}{g^{2}}\right\}\left\{-1, c \delta_{1} \delta_{2}(e+f \sqrt{d})\right\},
\end{aligned}
$$


where $\delta_{1}\left|u+w, \delta_{2}\right| k, \delta_{i} \in \mathbb{N},(x, y, z)=(a, b, c)$ is a relatively prime solution of $(2.10)$ in $\mathbb{N}$, and $(x, y, z)=(e, f, t)$ is a relatively prime solution of $(2.11)$ in $\mathbb{N}$. Then $\beta \in K_{2} O_{F}$ and $\beta^{2}=\{-1, m(u+\sqrt{d})\}$.

In particular, $\varepsilon>0$. We can take $k=1$ in $(2.10)$ and set

$$
\begin{aligned}
\beta= & \left\{-\frac{u+w+\sqrt{d}}{w}, \frac{2(u+w)(u+\sqrt{d})}{w^{2}}\right\} \\
& \times\left\{-\frac{b \sqrt{d}}{a}, \frac{m(u+w) c^{2}}{a^{2}}\right\}\{-1, c \delta\},
\end{aligned}
$$

where $\delta \mid u+w, \delta \in \mathbb{N}$, and $(x, y, z)=(a, b, c)$ is a relatively prime solution of $(2.10)$ in $\mathbb{N}$ with $k=1$. Then $\beta \in K_{2} O_{F}$ and $\beta^{2}=\{-1, m(u+\sqrt{d})\}$.

With the preceding method, we can also discuss an imaginary quadratic field $E=\mathbb{Q}(\sqrt{-d})$ to get results of [13] and the forms of elements of order 4 of $\mathrm{K}_{2} \mathrm{O}_{\mathrm{E}}$.

Theorem 2.3 ([13], Theorems 3.10 and 3.13). Let $F=\mathbb{Q}(\sqrt{d}), E=$ $\mathbb{Q}(\sqrt{-d}), d>2$ a squarefree integer, and $m$ an odd positive divisor of $d$.

(1) There is $\beta \in K_{2} O_{E}$ with $\beta^{2}=\{-1, m\}$ if and only if $\varepsilon m \in N F$, where $\varepsilon \in\{1,2\}$.

(2) If $-d=u^{2}-2 w^{2}, u, w \in \mathbb{N}$, then there is $\beta \in K_{2} O_{E}$ with $\beta^{2}=$ $\{-1, m(u+\sqrt{-d})\}$ if and only if $m(u+w) \in N F$.

Similarly, suppose $m \mid d, \varepsilon m \in N F$, and set

$$
\beta=\left\{-\frac{b \sqrt{-d}}{a}, \frac{\varepsilon m c^{2}}{a^{2}}\right\}\{-1, c\},
$$

where $(x, y, z)=(a, b, c)$ is a relatively prime solution of $\varepsilon z^{2}=x^{2}-d y^{2}$ in $\mathbb{N}$. Then $\beta \in K_{2} O_{E}$ and $\beta^{2}=\{-1, m\}$.

Suppose $m \mid d,-d=u^{2}-2 w^{2}, u, w \in \mathbb{N}, m(u+w) \in N F$, and set

$$
\begin{aligned}
\beta= & \left\{-\frac{u+w+\sqrt{-d}}{w}, \frac{2(u+w)(u+\sqrt{-d})}{w^{2}}\right\} \\
& \times\left\{-\frac{b \sqrt{-d}}{a}, \frac{m(u+w) c^{2}}{a^{2}}\right\}\{-1, c \delta\},
\end{aligned}
$$

where $\delta \mid u+w, \delta \in \mathbb{N}$, and $(x, y, z)=(a, b, c)$ is a relatively prime solution of $m(u+w) z^{2}=x^{2}-d y^{2}$ in $\mathbb{N}$. Then $\beta \in K_{2} O_{E}$ and $\beta^{2}=\{-1, m(u+\sqrt{-d})\}$.

3. Real quadratic fields. To investigate whether $\varepsilon>0$ in (2.6) and (2.12), we divide them into two cases. 
Definition 3.1. Let $F=\mathbb{Q}(\sqrt{d}), d>2$ a squarefree integer. Set

$S_{0}=\{m \mid m$ is an odd positive divisor of $d\}$,

$S_{1}=\left\{\varepsilon m \mid m \in S_{0}\right.$ and $(m, \varepsilon), \varepsilon>0$, satisfies (2.6) or (2.12)\},

$S_{2}=\left\{|\varepsilon| m \mid m \in S_{0}\right.$ and $(m, \varepsilon), \varepsilon<0$, satisfies (2.6) or (2.12),

but $\left.m, 2 m \notin S_{1}\right\}$.

In [17], we give the relation between $S_{1}$ and $C(E)$ (the narrow class group of the field $E=\mathbb{Q}(\sqrt{-d}))$. In fact, if -1 or -2 is in $N F$, then $S_{2}=\emptyset$; if $d \equiv-1 \bmod 8$, then $S_{2}=\emptyset$ by the quadratic reciprocity law or by [17], Lemma 3.4. Below, we explain why $S_{2} \neq \emptyset$.

LEMMA 3.1. Let $\pi: B \rightarrow(c)$ be a surjective homomorphism of a finite Abelian p-group $B$. If $b \in B$ is an element of minimal order such that $\pi(b)=c$, then there exists a subgroup $B^{\prime}$ of $B$ satisfying $B=(b) \times B^{\prime}$ and $\pi\left(B^{\prime}\right) \subset\left(c^{p}\right)$.

Proof. Since $B$ is Abelian, we have $B=\left(b_{1}\right) \times \ldots \times\left(b_{t}\right)$. From the surjectivity of $\pi$ it follows that $\left(\pi\left(b_{j}\right)\right)=(c)$ for some $j$. We assume that $b_{1}$ is an element of minimal order among all $b_{j}$ satisfying $\left(\pi\left(b_{j}\right)\right)=(c)$; we can also assume that $\pi\left(b_{1}\right)=c$, because $b_{1}$ can be replaced by some power of $b_{1}$ if necessary.

For $i \geq 2$, if $\left(\pi\left(b_{i}\right)\right)=(c)$, i.e., $\pi\left(b_{i}\right)=c^{t}$ with $p \nmid t$, then we take $b_{i}^{\prime}=b_{1}^{p-t} b_{i}$. If $\left(\pi\left(b_{i}\right)\right) \neq(c)$, i.e., $\left(\pi\left(b_{i}\right)\right) \subset\left(c^{p}\right)$, then we take $b_{i}^{\prime}=b_{i}$. Then the group $B^{\prime}$ generated by $b_{2}^{\prime}, \ldots, b_{t}^{\prime}$ satisfies $B=\left(b_{1}\right) \times B^{\prime}$ and $\pi\left(B^{\prime}\right) \subset\left(c^{p}\right)$.

Now let $x \in B$ be an element of minimal order satisfying $\pi(x)=c$. Then $x=b_{1}^{j} b^{\prime}$ with $b^{\prime} \in B^{\prime}$. Consequently, $c=\pi(x)=\pi\left(b_{1}\right)^{j} \pi\left(b^{\prime}\right)=c^{j} c^{p k}$ for some $k \in \mathbb{Z}$. Hence $p \nmid j$.

From $1=x^{o(x)}=b_{1}^{j o(x)} b^{\prime o(x)}$, we get $b_{1}^{j o(x)} \in B^{\prime}$, and hence $b_{1}^{o(x)}=1$. Therefore $o\left(b_{1}\right) \leq o(x)$. On the other hand, $o(x) \leq o\left(b_{1}\right)$, by the minimality of $o(x)$. It follows that $o(x)=o\left(b_{1}\right)$, and consequently $B=(x) \times B^{\prime}$.

TheOREM 3.1. Let $F=\mathbb{Q}(\sqrt{d}), d>2$ squarefree.

(1) If $d \not \equiv \pm 1 \bmod 8$, then $\left(K_{2} O_{F}\right)_{2}=\left(\alpha_{1}\right) \times\left(\alpha_{2}\right) \times H$, where $\alpha_{1}=$ $\{-1,-1\}, H \subset \Re_{2} F$, and $\alpha_{2}$ is an element of minimal order of $\left(\mathrm{K}_{2} \mathrm{O}_{F}\right)_{2}$ with Hilbert symbol $\eta_{\infty_{i}}\left(\alpha_{2}\right)=(-1)^{i}, \infty_{i}$ real places, $i=1,2$.

(2) If $d \equiv 1 \bmod 8$, then $\left(K_{2} O_{F}\right)_{2}=\left(\alpha_{1}\right) \times\left(\alpha_{2}\right) \times\left(\alpha_{3}\right) \times H$, where $\alpha_{1}=\{-1,-1\}, H \subset \Re_{2} F$, and $\alpha_{2}, \alpha_{3}$ are elements of minimal order of $K_{2} O_{F}$ satisfying $\eta_{\infty_{i}}\left(\alpha_{2}\right)=(-1)^{i}, \eta_{\infty_{i}}\left(\alpha_{3}\right)=1, \eta_{P_{i}}\left(\alpha_{3}\right)=-1, P_{i} \mid 2$, $i=1,2$; moreover, either $\alpha_{2}$ or $\alpha_{3}$ is an element of order 2 .

(3) If $d \equiv-1 \bmod 8$, then $\left(K_{2} O_{F}\right)_{2}=\left(\alpha_{1}\right) \times\left(\alpha_{2}\right) \times H$, where $\alpha_{1}=$ $\{-1,-1\}$ and $\alpha_{2}$ is an element of minimal order of $K_{2} O_{F}$ satisfying $\eta_{\infty_{i}}(\alpha)$ $=(-1)^{i}, i=1,2$. Moreover $\alpha_{2}$ is of order at least 8 . 
Proof. By [2], Theorem 2, or [10], $\S 15$, we obtain the commutative diagram with exact rows and columns:

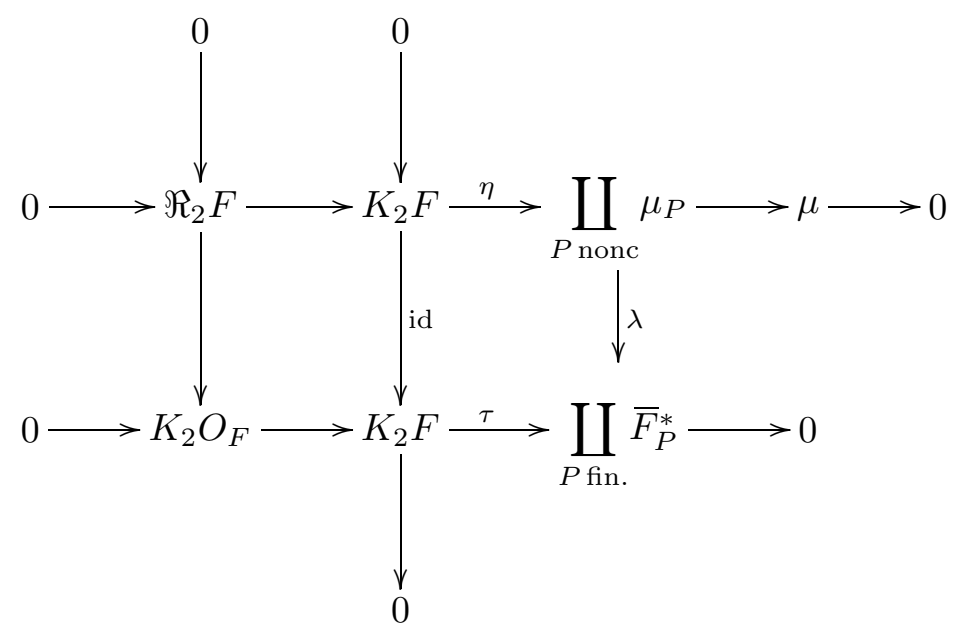

where the homomorphism $\lambda$ is defined as follows:

$$
\begin{gathered}
\lambda: \coprod_{P \text { nonc }} \mu_{P} \rightarrow \coprod_{P \text { fin. }} \bar{F}_{P}^{*}, \\
\lambda\left(a_{P}\right)= \begin{cases}1 & \text { if } P \text { is real }, \\
a_{P}^{m_{P} /(N P-1)} & \text { if } P \text { is finite },\end{cases}
\end{gathered}
$$

where $\mu_{P}$ is the group of roots of unity in the local completion field $F_{P}$, $a_{P} \in \mu_{P}, m_{P}=\left|\mu_{P}\right|, \bar{F}_{P}$ is the residue class field of the completion field $F_{P}$, and $N P=\left|\bar{F}_{P}\right|$.

By diagram chase, we get the exact sequence

$$
0 \rightarrow \Re_{2} F \rightarrow K_{2} O_{F} \stackrel{\eta}{\rightarrow} \operatorname{Im} \eta \cap \operatorname{ker} \lambda \rightarrow 0 .
$$

Since the group $K_{2} O_{F}$ is finite, we obtain the exact sequence of their 2-Sylow subgroups

$$
0 \rightarrow\left(\Re_{2} F\right)_{2} \rightarrow\left(K_{2} O_{F}\right)_{2} \stackrel{\eta}{\rightarrow}(\operatorname{Im} \eta \cap \operatorname{ker} \lambda)_{2} \rightarrow 0 .
$$

If $d \equiv-3 \bmod 9$, then $m_{P}=3(N P-1)$ for $P$ a place over 3 and $m_{P}=$ $N P-1$ for all $P \notin S$ and $P \nmid 3$; otherwise $m_{P}=N P-1$ for $P \notin S$. Therefore $(\operatorname{Im} \eta \cap \operatorname{ker} \lambda)_{2}=\operatorname{Im} \eta \cap\left(\mu_{\infty_{1}} \times \mu_{\infty_{2}} \times \coprod_{P \mid 2} \mu_{P}^{N P-1}\right)$.

(1) If $d \not \equiv \pm 1 \bmod 8$, then

$$
\left(K_{2} O_{F}\right)_{2} /\left(\Re_{2} F\right)_{2} \cong \operatorname{Im} \eta \cap\left(\mu_{\infty_{1}} \times \mu_{\infty_{2}} \times \mu_{P}^{N P-1}\right) \cong \mathbb{Z} /(2) \oplus \mathbb{Z} /(2),
$$

where $P \mid 2$ and $\mu_{\infty_{i}}=\mu_{P}^{N P-1}=\{ \pm 1\}$. Since $\eta\left(\alpha_{1}\right)=\eta(\{-1,-1\})=\beta_{1}=$ $(-1,-1,1)$ and $\beta_{2}=(-1,1,-1)$ are two generators of $(\operatorname{Im} \eta \cap \operatorname{ker} \lambda)_{2}$ we have $\left(K_{2} O_{F}\right)_{2}=\left(\alpha_{1}\right) \times \eta^{-1}\left(\beta_{2}\right)$, so we get $\alpha_{2}$ by Lemma 3.1. 
(2) If $d \equiv 1 \bmod 8$, then

$$
\begin{aligned}
\left(K_{2} O_{F}\right)_{2} /\left(\Re_{2} F\right)_{2} & \cong \operatorname{Im} \eta \cap\left(\mu_{\infty_{1}} \times \mu_{\infty_{2}} \times \mu_{P_{1}} \times \mu_{P_{2}}\right) \\
& \cong \mathbb{Z} /(2) \oplus \mathbb{Z} /(2) \oplus \mathbb{Z} /(2),
\end{aligned}
$$

where $P_{i} \mid 2$ and $\mu_{P_{i}}=\{ \pm 1\}, i=1,2$. Since $\eta\left(\alpha_{1}\right)=\eta(\{-1,-1\})=\beta_{1}=$ $(-1,-1,-1,-1), \beta_{2}=(1,-1,1,-1), \beta_{3}=(1,1,-1,-1)$ are three generators of $(\operatorname{Im} \eta \cap \operatorname{ker} \lambda)_{2}$, we have $\left(K_{2} O_{F}\right)_{2}=\left(\alpha_{1}\right) \times \eta^{-1}\left\{\beta_{2}, \beta_{3}\right\}$, where $\alpha_{1}=\{-1,-1\}$.

Suppose $-1 \in N F$. Take $\alpha_{2}=\{-1, u+\sqrt{d}\}$, where $u^{2}+w^{2}=d, u, w \in$ $\mathbb{N}$. Hence $\eta\left(\alpha_{2}\right)=\beta_{2}$, so we get $\alpha_{3}$ by Lemma 3.1.

Suppose $-1 \notin N F$. There is a prime divisor $p \equiv 3 \bmod 4$ of $d$. Take $\alpha_{3}=\{-1, p\}$, so we also get $\alpha_{2}$ by Lemma 3.1 .

(3) If $d \equiv-1 \bmod 8$, then

$$
\left(K_{2} O_{F}\right)_{2} /\left(\Re_{2} F\right)_{2} \cong \operatorname{Im} \eta \cap\left(\mu_{\infty_{1}} \times \mu_{\infty_{2}} \times \mu_{P}\right) \cong \mathbb{Z} /(2) \oplus \mathbb{Z} /(4),
$$

where $\mu_{P}=\{ \pm 1, \pm i\}$ and $P \mid 2$. As $\eta\left(\alpha_{1}\right)=\eta(\{-1,-1\})=\beta_{1}=(-1,-1,1)$ and $\beta_{2}=(-1,1, i)$ are two generators of $(\operatorname{Im} \eta \cap \operatorname{ker} \lambda)_{2}$, we have $\left(K_{2} O_{F}\right)_{2}=$ $\left(\alpha_{1}\right) \times \eta^{-1}\left(\beta_{2}\right)$. By [3], Theorem 2, we know that $r_{2}\left(K_{2} O_{F}\right)-r_{2}\left(\Re_{2} F\right)=1$ and $\alpha_{1}=\{-1,-1\} \notin \Re_{2} F$. Therefore, we get $\alpha_{2}$ by Lemma 3.1, which is of order at least 8 .

In Theorem 3.1, if $\alpha_{2}$ and $\alpha_{3}$ are elements of minimal order of $K_{2} O_{F}$, then there are also direct decompositions for $\mathrm{K}_{2} \mathrm{O}_{F}$.

Corollary 3.1. Let $F=\mathbb{Q}(\sqrt{d}), d>2$ a squarefree integer.

(1) If $S_{2} \neq \emptyset$, then $\alpha_{2}$ must be of order 4 in Theorem 3.1 and $r_{4}\left(K_{2} O_{F}\right)$ $=r_{4}\left(\Re_{2} F\right)+1$.

(2) If $S_{2}=\emptyset$ and $-1,-2 \notin N F$, then there is an 8-order element in $\mathrm{K}_{2} \mathrm{O}_{\mathrm{F}}$.

Proof. (1) Since $S_{2} \neq \emptyset$ and $-1,-2 \notin N F$, we have $d \not \equiv-1 \bmod 8$ and $o\left(\alpha_{2}\right)>2$ in Theorem 3.1 by [3], Theorem 2. Also $S_{2} \neq \emptyset$ implies that $o\left(\alpha_{2}\right) \leq 4$ by (2.7) or (2.13). Therefore, $\alpha_{2}$ must be of order 4 and $r_{4}\left(K_{2} O_{F}\right)=r_{4}\left(\Re_{2} F\right)+1$.

(2) Since $-1,-2 \notin N F$, we have $o\left(\alpha_{2}\right)>2$ by [3], Theorem 2. Since $S_{2}=\emptyset$, also $o\left(\alpha_{2}\right)>4$ by (2.8) or (2.14). Therefore, $\alpha_{2}$ is of order at least 8 .

TheOREM 3.2. Let $F=\mathbb{Q}(\sqrt{d}), d>2$ a squarefree integer, $d \equiv 1 \bmod 8$, $-1 \in N F$, and $2 \notin N F$. Then $o\left(\alpha_{3}\right)=4$ in Theorem 3.1 if and only if there is an equation $\varepsilon m z^{2}=x^{2}+d y^{2}$ with $m \in S_{0}, m \neq 1, d$, and $\varepsilon \in\{1,2\}$, which has a relatively prime solution $(x, y, z)=(a, b, c)$ in $\mathbb{N}$ such that either $m \equiv 1 \bmod 8$ and $c \equiv 3 \bmod 4$, or $m \equiv 5 \bmod 8$ and $c \equiv 1 \bmod 4$.

Proof. Since $-1 \in N F$ and $2 \notin N F$, we have $o\left(\alpha_{3}\right)>2$. Suppose that $\varepsilon m \in S_{1}, m \neq 1, d, \varepsilon \in\{1,2\}$, i.e., the Diophantine equation $\varepsilon m z^{2}=$ 
$x^{2}+d y^{2}$ has a relatively prime solution $(x, y, z)=(a, b, c)$ in $\mathbb{N}$. Then $\beta=$ $\left\{-\frac{b \sqrt{d}}{a}, \frac{\varepsilon m c^{2}}{a^{2}}\right\}\{-1, c\} \in K_{2} O_{F}$ and $\beta^{2}=\{-1, m\}$. Now, we discuss whether $\eta_{P_{i}}(\beta)=-1, P_{i} \mid 2, i=1,2$. Since $d \equiv 1 \bmod 8$, the local field $Q_{2}(\sqrt{d}) \cong$ $Q_{2}$. In the local field $Q_{2}$, we compute the value of the Hilbert symbols $\left[-\frac{b \sqrt{d}}{a}, \frac{\varepsilon m c^{2}}{a^{2}}\right]_{2}$.

(i) If $\varepsilon=2$ and $m \equiv 1 \bmod 8$, then $a, b, c$ are odd and $-b \sqrt{d} / a$ is a solution of the equation

$$
X^{2}=\varepsilon m c^{2} / a^{2}-1 .
$$

Since $\varepsilon m c^{2} / a^{2}-1 \equiv 1 \bmod 16$, the equation (3.17) has two solutions $\gamma \equiv 1$ or 7 mod 8 by the Hensel lemma. By the table in [16], p. 250, $\left[-b \sqrt{d} / a, \varepsilon m c^{2} / a^{2}\right]_{2}$ $=[\gamma, 2]_{2}=1$.

(ii) If $\varepsilon=2$ and $m \equiv 5 \bmod 8$, then $a, b, c$ are all odd, so $\varepsilon m c^{2} / a^{2}-1 \equiv$ $9 \bmod 16$. Hence the equation (3.17) has two solutions $\gamma \equiv 3$ or $5 \bmod 8$ by the Hensel lemma. By the table in [16], p. 250, $\left[-b \sqrt{d} / a, \varepsilon m c^{2} / a^{2}\right]_{2}=$ $[\gamma, 2]_{2}=-1$.

(iii) If $\varepsilon=1, m \equiv 1 \bmod 8$ and $\varepsilon m c^{2} / a^{2} \in Q_{2}^{2}$, then $\left[-b \sqrt{d} / a, \varepsilon m c^{2} / a^{2}\right]_{2}$ $=1$.

(iv) If $\varepsilon=1, m \equiv 5 \bmod 8$, then $a$ or $b \equiv 2 \bmod 4$, and $c$ is odd. Hence, by the table in [16], p. $250,\left[-b \sqrt{d} / a, \varepsilon m c^{2} / a^{2}\right]_{2}=[\gamma, 5]_{2}=-1$, where $\gamma \equiv 2$ or $6 \bmod 8$.

Therefore

$$
\left[-\frac{b \sqrt{d}}{a}, \frac{\varepsilon m c^{2}}{a^{2}}\right]_{2}= \begin{cases}1 & \text { if } m \equiv 1 \bmod 8 \\ -1 & \text { if } m \equiv 5 \bmod 8 .\end{cases}
$$

By the same table,

$$
[-1, c]_{2}= \begin{cases}1 & \text { if } c \equiv 1 \bmod 4 \\ -1 & \text { if } c \equiv 3 \bmod 4 .\end{cases}
$$

In Theorem 3.1, $\alpha_{3}=\beta$, i.e., $\eta_{P_{i}}(\beta)=-1, P_{i} \mid 2, i=1,2$, if and only if either $m \equiv 1 \bmod 8$ and $c \equiv 3 \bmod 4$, or $m \equiv 5 \bmod 8$ and $c \equiv 1 \bmod 4$.

Suppose that $(x, y, z)=\left(a^{\prime}, b^{\prime}, c^{\prime}\right)$ is another relatively prime solution of the equation $\varepsilon m z^{2}=x^{2}+d y^{2}$ in $\mathbb{N}$ with $c^{\prime} \equiv c+2 \bmod 4$. We can also get $\beta^{\prime}$ by (2.7). But $\eta_{P_{i}}(\beta)=-\eta_{P_{i}}\left(\beta^{\prime}\right)$, i.e., $\eta_{P_{i}}\left(\beta \beta^{\prime}\right)=-1, P_{i} \mid 2, i=1$, 2. So $\alpha_{3}=\beta \beta^{\prime}$ must be of order 2 in $K_{2} O_{F}$ in contradiction with the assumption. Hence we obtain

Corollary 3.2. Let $d=p_{1} \ldots p_{r+s} \equiv 1 \bmod 8$, with each prime $p_{i} \equiv 1$ $\bmod 4, r, s \geq 1$, and some prime $p_{i} \equiv 5 \bmod 8$. If the Diophantine equation $\varepsilon m z^{2}=x^{2}+d y^{2}, \varepsilon \in\{1,2\}, m=p_{1} \ldots p_{r}$, has a non-trivial solution in $\mathbb{Z}$, then for every relatively prime solution $(x, y, z)=(a, b, c)$ of this equation in $\mathbb{N}$ we have $c \equiv 1$ or $3 \bmod 4$. 
If $d=p_{1} \ldots p_{r} \equiv 1 \bmod 8$, with each prime $p_{i} \equiv 1 \bmod 8$, and $u^{2}-2 w^{2}=$ $d, u, w \in \mathbb{N}, w \equiv 0 \bmod 4, u \equiv 1 \bmod 4$, we can also get a result similar to Corollary 3.2.

Next, we investigate the property of $c \equiv 1$ or $3 \bmod 4$. In particular, if $F=\mathbb{Q}(\sqrt{d}), d=p_{1} p_{2}$, with each prime $p_{i} \equiv 5 \bmod 8$, we get:

Lemma 3.2. Let $F=\mathbb{Q}\left(\sqrt{p_{1} p_{2}}\right), E=\mathbb{Q}\left(\sqrt{-p_{1} p_{2}}\right)$, with each prime $p_{i} \equiv 5 \bmod 8$. By the Legendre theorem the Diophantine equation

$$
x^{2}+p_{1} p_{2} y^{2}=\varepsilon p_{1} z^{2}, \quad \varepsilon \in\{1,2\},
$$

has a relatively prime solution $(x, y, z)=(a, b, c)$ in $\mathbb{N}$. Then $c \equiv 1 \bmod 4$ if and only if $16 \mid h\left(-p_{1} p_{2}\right)$, which is the class number of the field $E=$ $\mathbb{Q}\left(\sqrt{-p_{1} p_{2}}\right)$; in other words, $c \equiv 3 \bmod 4$ if and only if $8 \| h\left(-p_{1} p_{2}\right)$.

Proof. By genus theory, $r_{2}(C(E))=2$, where $C(E)$ is the class group of $E$. If $\left(\frac{p_{2}}{p_{1}}\right)=1$, the Diophantine equation $x^{2}+p_{1} p_{2} y^{2}=p_{1} z^{2}$ is solvable in $\mathbb{Z}$; if $\left(\frac{p_{2}}{p_{1}}\right)=-1$, the Diophantine equation $x^{2}+p_{1} p_{2} y^{2}=2 p_{1} z^{2}$ is solvable in $\mathbb{Z}$.

Let $P$ be an ideal of $E$ with $P^{2}=\varepsilon p_{1} O_{E}$. Since (3.18) has a relatively prime solution $(x, y, z)=(a, b, c)$ in $\mathbb{N}$, we have $\left(a+b \sqrt{-p_{1} p_{2}}\right) O_{E}=P C^{2}$, where $C \bar{C}=c O_{E}, \bar{C}$ a conjugate ideal of $C$. Hence $[P]=[C]^{2} \in C^{2}(E)$, so $8 \mid h\left(-p_{1} p_{2}\right)$ by genus theory. It is clear that

$$
\left(\frac{-p_{1} p_{2}}{c}\right)=\left(\frac{-1}{c}\right)\left(\frac{c}{p_{1}}\right)\left(\frac{c}{p_{2}}\right)=1
$$

by (3.18).

Assume that $c \equiv 1 \bmod 4$, i.e.,

$$
\begin{aligned}
\left(\frac{-1}{c}\right)=1 \\
\Leftrightarrow\left(\frac{c}{p_{1}}\right)\left(\frac{c}{p_{2}}\right)=1 \text {, i.e., }\left(\frac{c}{p_{1}}\right)=\left(\frac{c}{p_{2}}\right) \\
\Leftrightarrow \quad \text { the Diophantine equation } \varepsilon^{\prime} c z^{2}=x^{2}+p_{1} p_{2} y^{2}, \varepsilon^{\prime} \in\{1,2\}, \text { is solvable } \\
\quad \text { in } \mathbb{Z} \\
\Leftrightarrow \quad N_{F / \mathbb{Q}}\left(P^{\prime} C\right) \in N F, \text { where } P^{\prime} \text { is an ideal of } E \text { such that } P^{\prime 2}=\varepsilon^{\prime} O_{E}, \\
\quad \text { by the Gauss theorem } \\
\Leftrightarrow \quad\left[P^{\prime} C\right] \in C^{2}(E), \text { i.e., }[C]^{2} \in C^{4}(E) \\
\Leftrightarrow \quad 16 \mid h\left(-p_{1} p_{2}\right) .
\end{aligned}
$$$$
\Leftrightarrow N_{F / \mathbb{Q}}\left(P^{\prime} C\right) \in N F \text {, where } P^{\prime} \text { is an ideal of } E \text { such that } P^{\prime 2}=\varepsilon^{\prime} O_{E} \text {, }
$$

Hence, Lemma 3.2 follows. 
Theorem 3.3. Let $F=\mathbb{Q}\left(\sqrt{p_{1} p_{2}}\right), E=\mathbb{Q}\left(\sqrt{-p_{1} p_{2}}\right)$, with each prime $p_{i} \equiv 5 \bmod 8$. Then $\left(K_{2} O_{F}\right)_{2} \cong \mathbb{Z} /(2) \times \mathbb{Z} /(2) \times \mathbb{Z} /(4)$ if and only if $16 \mid h\left(-p_{1} p_{2}\right)$; in other words, $K_{2} O_{F}$ has an element of order 8 if and only if $8 \| h\left(-p_{1} p_{2}\right)$.

Proof. This follows from Lemma 3.2 and Theorem 3.2.

Example 1: $F=\mathbb{Q}(\sqrt{5 \cdot 13})$. Since the Diophantine equation $x^{2}+$ $5 \cdot 13 y^{2}=10 z^{2}$ has a solution $(x, y, z)=(5,1,3)$, we have $8 \| h(-5 \cdot 13)$ and $K_{2} O_{F}$ has an element of order 8 by Lemma 3.2 and Theorem 3.2.

Example 2: $F=\mathbb{Q}(\sqrt{5 \cdot 37})$. Since the Diophantine equation $x^{2}+$ $5 \cdot 37 y^{2}=10 z^{2}$ has a solution $(x, y, z)=(25,1,9)$, we have $\left(K_{2} O_{F}\right)_{2} \cong$ $\mathbb{Z} /(2) \oplus \mathbb{Z} /(2) \oplus \mathbb{Z} /(4)$ and $16 \mid h(-5 \cdot 37)$.

TheOREM 3.4. Let $F=\mathbb{Q}(\sqrt{d}), d>2$ a squarefree integer, $d \equiv-1 \bmod 8$, and $2 \notin N F$. Then

$$
r_{4}\left(K_{2} O_{F}\right)= \begin{cases}r_{4}\left(\Re_{2} F\right)+1 & \text { if } \varepsilon m \in S_{1}, m \equiv \pm 3 \bmod 8 \\ r_{4}\left(\Re_{2} F\right) & \text { otherwise. }\end{cases}
$$

Moreover, in the second case, there is an element of order 16 in $\mathrm{K}_{2} \mathrm{O}_{F}$.

Proof. Since $d \equiv-1 \bmod 8$, we have $S_{2}=\emptyset$ by [17], Lemma 3.4. If $\varepsilon m \in S_{1}$, then $\beta=\left\{-\frac{b \sqrt{d}}{a}, \frac{\varepsilon m c^{2}}{a^{2}}\right\}\{-1, c\} \in K_{2} O_{F}$ and $\beta^{2}=\{-1, m\}$, where $(x, y, z)=(a, b, c)$ is a relatively prime solution of $\varepsilon m z^{2}=x^{2}+d y^{2}$ in $\mathbb{N}$.

In the completion field $F_{P} \cong Q_{2}(i), P \mid 2$, we have $\eta_{P}\{-1, c\}=[-1, c]_{P}$ $=1$ by the Artin-Hasse theorem [11]. Let $\delta=-b \sqrt{d} / a, \varepsilon m b^{2} / a^{2}=1+\delta^{2}$. Then

$$
\begin{aligned}
\eta_{P}(\beta) & =\left[\delta, 1+\delta^{2}\right]_{P}[-1, c]_{P}=[\delta,(1+i \delta)(1-i \delta)]_{P} \\
& =\left[1+\delta^{2}, i\right]_{P}[1+i \delta,-1]_{P}=[\varepsilon m, i]_{P}[a+b i \sqrt{d},-1]_{P} .
\end{aligned}
$$

Since $d \equiv-1 \bmod 8$, we have $a+b i \sqrt{d} \in Q_{2}$. By the Artin-Hasse theorem, $[-1, a+b i \sqrt{d}]_{P}=1$ and

$$
[i, 2 m]_{P}=[i, m]_{P}=i^{\left(m^{2}-1\right) / 4}= \begin{cases}1 & \text { if } m \equiv \pm 1 \bmod 8 \\ -1 & \text { if } m \equiv \pm 3 \bmod 8 .\end{cases}
$$

Therefore, $\beta \in \Re_{2} F$ if and only if $m \equiv \pm 1 \bmod 8$. By Theorem 3.1, we get the assertion of Theorem 3.4.

By Theorem 3.4, we get the following result: if $F=\mathbb{Q}(\sqrt{d}), d=p q, p, q$ prime, $p \equiv-q \equiv 3 \bmod 8$, then $\left(K_{2} O_{F}\right)_{2} \cong \mathbb{Z} /(2) \oplus \mathbb{Z} /(8)$, which is proved in another way in [4]. On the other hand, we can generalize it.

Theorem 3.5. Let $F=\mathbb{Q}(\sqrt{d}), d>2$ a squarefree integer, $d \equiv-1 \bmod$ 8 , and $2 \notin N F$. Suppose that $d=p q r$, where $p, q, r$ are primes, i.e., $(p, q, r) \equiv(1,3,5)$ or $(7,5,5)$ or $(7,3,3) \bmod 8$. 
(1) If $\left(\frac{q}{p}\right)=\left(\frac{r}{p}\right)=1$, then $\left(K_{2} O_{F}\right)_{2} \cong \mathbb{Z} /\left(2^{i}\right) \oplus \mathbb{Z} /\left(2^{j}\right) \oplus \mathbb{Z} /(2)$, where $i \geq 3, j \geq 2$.

(2) If $\left(\frac{q}{p}\right)=\left(\frac{r}{p}\right)=-1$, then $\left(K_{2} O_{F}\right)_{2} \cong \mathbb{Z} /\left(2^{i}\right) \oplus \mathbb{Z} /(2) \oplus \mathbb{Z} /(2)$, where $i \geq 4$.

(3) If $\left(\frac{q}{p}\right) \neq\left(\frac{r}{p}\right)$, then $\left(K_{2} O_{F}\right)_{2} \cong \mathbb{Z} /(8) \oplus \mathbb{Z} /(2) \oplus \mathbb{Z} /(2)$.

Proof. (1) If $\left(\frac{q}{p}\right)=\left(\frac{r}{p}\right)=1, r_{4}\left(K_{2} O_{F}\right)=2$ by the tables of [14]. We get the result by Theorem 3.1.

(2) If $\left(\frac{q}{p}\right)=\left(\frac{r}{p}\right)=-1$, then $r_{4}\left(K_{2} O_{F}\right)=1$ by the tables of [14]. In fact, if $(p, q, r) \equiv(1,3,5)$ or $(7,5,5) \bmod 8$, then $(m, \varepsilon)=(p, 2)$ satisfies $(2.6)$; if $(p, q, r) \equiv(7,3,3) \bmod 8$, then $(m, \varepsilon)=(p, 1)$ satisfies $(2.6)$. By Theorem 3.4, we get $r_{4}\left(K_{2} O_{F}\right)=r_{4}\left(\Re_{2} F\right)=1$ and $o\left(\alpha_{2}\right) \geq 16$ in Theorem 3.1.

(3) If $\left(\frac{q}{p}\right) \neq\left(\frac{r}{p}\right)$, then $r_{4}\left(K_{2} O_{F}\right)=1$ by the tables of [14]. There is $(m, \varepsilon)$ with $m \equiv \pm 3 \bmod 8$ and $\varepsilon \in\{1,2\}$ satisfying (2.6). By Theorem 3.4, we get $r_{4}\left(K_{2} O_{F}\right)=r_{4}\left(\Re_{2} F\right)+1=1$ and $o\left(\alpha_{2}\right)=8$ in Theorem 3.1.

4. Imaginary quadratic fields. In this section, we consider imaginary quadratic fields $E=\mathbb{Q}(\sqrt{-d}), d>2$ a squarefree integer. By [15], we have $\left[\Delta: E^{*}\right]=4$, where $\Delta=\left\{z \in E^{*} \mid\{-1, z\}=1\right\}$ is called the Tate kernel. Since $2 \in \Delta$, we have

$$
\Delta=E^{* 2} \cup 2 E^{* 2} \cup \delta E^{* 2} \cup 2 \delta E^{* 2} .
$$

Below, we find such elements $\delta \in \Delta$ for some imaginary quadratic fields.

From [2], we know the following relation between $K_{2} O_{E}$ and $\Re_{2} E$ (the Hilbert kernel of $E$ ):

$$
\left(K_{2} O_{E} / \Re_{2} E\right)_{2} \cong \begin{cases}0 & \text { if } d \neq \pm 1 \bmod 8 \\ \mathbb{Z} /(2) & \text { if } d \equiv-1 \bmod 8, \\ \mathbb{Z} /(2) & \text { if } d \equiv 1 \bmod 8\end{cases}
$$

If $d \equiv-1 \bmod 8$, then there is a prime divisor $p$ of $d$ with $p \equiv 3 \bmod 4$. Hence $\alpha=\{-1, p\} \in K_{2} O_{E}$, but $\alpha \notin \Re_{2} E$, so $\left(K_{2} O_{E}\right)_{2} \cong(\alpha) \times\left(\Re_{2} E\right)_{2}$ by Lemma 3.1.

If $d \equiv 1 \bmod 8$, then $r_{2}\left(K_{2} O_{E}\right)=r_{2}\left(\Re_{2} E\right)$ by [3], Theorem 4. Hence, by Lemma 3.1, $\left(K_{2} O_{E}\right)_{2} \cong(\alpha) \times H$, where $\eta_{P}(\alpha)=-1, P \mid 2, o(\alpha) \geq 4$, and $H \subset\left(\Re_{2} E\right)_{2}$. Therefore, $r_{4}\left(K_{2} O_{E}\right)=r_{4}\left(\Re_{2} E\right)+1$ if $o(\alpha)=4$, and $r_{4}\left(K_{2} O_{E}\right)=r_{4}\left(\Re_{2} E\right)$ if $o(\alpha) \geq 8$.

Theorem 4.1. Let $E=\mathbb{Q}(\sqrt{-d}), F=\mathbb{Q}(\sqrt{d}), d>2$ a squarefree integer, $d \equiv 1 \bmod 8$, and $2 \notin N E$. Then $r_{4}\left(K_{2} O_{E}\right)=r_{4}\left(\Re_{2} E\right)+1$ if and only if there is an odd positive divisor $m \equiv \pm 3 \bmod 8$ of $d$ such that $\varepsilon m \in N F, \varepsilon \in\{1,2\}$.

Proof. By the preceding argument, Lemma 3.1, and (2.15), $r_{4}\left(K_{2} O_{E}\right)=$ $r_{4}\left(\Re_{2} E\right)+1$ if and only if there is $\beta=\left\{-\frac{b \sqrt{-d}}{a}, \frac{\varepsilon m c^{2}}{a^{2}}\right\}\{-1, c\} \notin \Re_{2} E$, 
where $m \mid d$ is positive and $(x, y, z)=(a, b, c)$ is a relatively prime solution of $\varepsilon m z^{2}=x^{2}-d y^{2}, \varepsilon \in\{1,2\}$, in $\mathbb{N}$. By the process of proving Theorem 3.4, we know that $\beta \notin \Re_{2} E$, i.e., $\eta_{P}(\beta)=-1, P \mid 2$, if and only if $m \equiv \pm 3 \bmod 8$.

By Theorem 4.1, we add some values to the tables in [13]:

Table 1

\begin{tabular}{|c|c|c|c|c|}
\hline$E$ & $p, q \bmod 8$ & $r_{4}$ & $r_{8}$ & $\delta$ \\
\hline \multirow{2}{*}{$\mathbb{Q}(\sqrt{-d})$} & 3,3 & 1 & 0 & -1 \\
\cline { 2 - 5 } & 5,5 & 1 & 0 & -1 \\
\hline
\end{tabular}

Table 2

\begin{tabular}{|c|c|c|c|c|c|}
\hline$E$ & $p, q, r$ mod 8 & The Legendre symbols & $r_{4}$ & $r_{8}$ & $\delta$ \\
\hline \multirow{4}{*}{$7,5,3$} & $\left(\frac{p}{q}\right)=\left(\frac{p}{r}\right)$ & 1 & 0 & $p$ \\
\hline & \multirow{4}{*}{$(\sqrt{-p q r})$} & otherwise & 1 & 0 & $-p$ \\
\cline { 3 - 6 } & \multirow{3}{*}{$1,5,5$} & $\left(\frac{q}{p}\right)=\left(\frac{r}{p}\right)=1$ & 2 & & \\
\hline & & $\left(\frac{q}{p}\right)=\left(\frac{r}{p}\right)=-1$ & 1 & 1 & \\
\hline & $\left(\frac{q}{p}\right) \neq\left(\frac{r}{p}\right)$ & 1 & 0 & -1 \\
\cline { 3 - 6 } & \multirow{3}{*}{$1,3,3$} & $\left(\frac{q}{p}\right)=\left(\frac{r}{p}\right)=1$ & 2 & & \\
\hline & & $\left(\frac{q}{p}\right)=\left(\frac{r}{p}\right)=-1$ & 1 & 1 & \\
\hline & $\left(\frac{q}{p}\right) \neq\left(\frac{r}{p}\right)$ & 1 & 0 & -1 \\
\hline
\end{tabular}

Proof. 1. For Table 1, we need to consider the case $(p, q) \equiv(5,5) \bmod 8$. If $\left(\frac{q}{p}\right)=1$, then $p, p q \in N F$; if $\left(\frac{q}{p}\right)=-1$, then $2 p, p q \in N F$. By the tables of [13] and Theorem 4.1, $r_{4}\left(K_{2} O_{E}\right)=r_{4}\left(\Re_{2} E\right)+1=1$ and $\{-1, p q\}=1$, i.e., $\{-1,-1\}=1$.

2. For Table 2:

The case $(p, q, r) \equiv(7,5,3) \bmod 8$. By the tables of [13], $r_{4}\left(K_{2} O_{E}\right)=1$. Suppose $\left(\frac{p}{q}\right)=\left(\frac{p}{r}\right)=1$ (similarly for $\left(\frac{p}{q}\right)=\left(\frac{p}{r}\right)=-1$ ). If $\left(\frac{r}{q}\right)=1$, then $q, p \in N F$; if $\left(\frac{r}{q}\right)=-1$, then $2 q, p \in N F$. Hence, by Theorem 4.1, $r_{4}\left(K_{2} O_{E}\right)=r_{4}\left(\Re_{2} E\right)+1=1$ and $\{-1, p\}=1$.

Suppose $\left(\frac{p}{q}\right)=-\left(\frac{p}{r}\right)=1$ (similarly for $\left(\frac{p}{q}\right)=-\left(\frac{p}{r}\right)=-1$ ). If $\left(\frac{r}{q}\right)=1$, then $q, q r \in N F$; if $\left(\frac{r}{q}\right)=-1$, then $2 q, q r \in N F$. Hence, by Theorem 4.1, $r_{4}\left(K_{2} O_{E}\right)=r_{4}\left(\Re_{2} E\right)+1=1$ and $\{-1, q r\}=1$, i.e., $\{-1,-p\}=1$.

The case $(p, q, r) \equiv(1,5,5) \bmod 8$. If $\left(\frac{q}{p}\right)=\left(\frac{r}{p}\right)=1$, then $r_{4}\left(K_{2} O_{E}\right)=2$ by the tables of [13].

If $\left(\frac{q}{p}\right)=\left(\frac{r}{p}\right)=-1$ then $r_{4}\left(K_{2} O_{E}\right)=1$ by the tables of [13] and $2 p, p q r \in$ $N F$. Hence $r_{4}\left(K_{2} O_{E}\right)=r_{4}\left(\Re_{2} E\right)=1$ and $r_{8}\left(K_{2} O_{E}\right)=1$ by Theorem 4.1. 
Suppose $\left(\frac{q}{p}\right)=-\left(\frac{r}{p}\right)=1$ (similarly for $\left(\frac{q}{p}\right)=-\left(\frac{r}{p}\right)=-1$ ). If $\left(\frac{r}{q}\right)=1$, then $q, p q r \in N F$; if $\left(\frac{r}{q}\right)=1$, then $2 q, p q r \in N F$. Hence, by the tables of [13] and Theorem 4.1, $r_{4}\left(K_{2} O_{E}\right)=r_{4}\left(\Re_{2} E\right)+1=1$ and $\{-1, p q r\}=1$, i.e., $\{-1,-1\}=1$.

The case $(p, q, r) \equiv(1,3,3) \bmod 8$. If $\left(\frac{q}{p}\right)=\left(\frac{r}{p}\right)=1$, then $r_{4}\left(K_{2} O_{E}\right)=2$ by the tables of [13].

If $\left(\frac{q}{p}\right)=\left(\frac{r}{p}\right)=-1$, then $r_{4}\left(K_{2} O_{E}\right)=1$ by the tables of [13] and $2 p, 2 p q r \in N F$. Hence, by Theorem 4.1, $r_{4}\left(K_{2} O_{E}\right)=r_{4}\left(\Re_{2} E\right)=1$ and $r_{8}\left(K_{2} O_{E}\right)=1$.

Suppose $\left(\frac{q}{p}\right)=-\left(\frac{r}{p}\right)=1$ (similarly for $\left(\frac{q}{p}\right)=-\left(\frac{r}{p}\right)=-1$ ). If $\left(\frac{q}{r}\right)=1$, then $2 q, 2 p q r \in N F$; if $\left(\frac{q}{r}\right)=-1$, then $q, 2 p q r \in N F$. Hence, by the tables of [13] and Theorem 4.1, $r_{4}\left(K_{2} O_{E}\right)=r_{4}\left(\Re_{2} E\right)+1=1$ and $\{-1,2 p q r\}=1$, i.e., $\{-1,-1\}=1$.

Acknowledgements. The author would like to thank Professors Feng Keqin and Qin Hourong for their helpful suggestions and many valuable conversations; he would also like to thank the referee for many corrections.

\section{References}

[1] B. Brauckmann, The 2-Sylow-subgroup of the tame kernel of number fields, Canad. J. Math. 43 (1991), 255-264.

[2] J. Browkin, The functor $K_{2}$ of the ring of integers of a number fields, in: Universal Algebra and Application, Banach Center Publ. 9, PWN, Warszawa, 1982, 187-195.

[3] J. Browkin and A. Schinzel, On Sylow 2-subgroups of $\mathrm{K}_{2} \mathrm{O}_{F}$ for quadratic fields $F$, J. Reine Angew. Math. 331 (1982), 104-113.

[4] A. Candiotti and K. Kramer, On the 2-Sylow subgroup of the Hilbert kernel of $K_{2}$ of number fields, Acta Arith. 52 (1989), 49-65.

[5] P. E. Conner and J. Hurrelbrink, The 4-rank of $\mathrm{K}_{2} \mathrm{O}_{F}$, Canad. J. Math. 41 (1989), 932-960.

[6] - - - On elementary abelian 2-Sylow $K_{2}$ of rings of integers of certain quadratic number fields, Acta Arith. 73 (1995), 59-65.

[7] E. Hecke, Lecture on the Theory of Algebraic Numbers, Grad. Texts in Math. 77, Springer, 1981.

[8] J. Hurrelbrink and M. Kolster, Tame kernels under relative quadratic extensions and Hilbert symbols, J. Reine Angew. Math. 499 (1998), 145-188.

[9] M. Kolster, The structure of the 2-Sylow subgroup of $K_{2}(O), I$, Comment. Math. Helv. 61 (1986), 576-588.

[10] J. Milnor, Introduction to Algebraic K-theory, Ann. of Math. Stud. 72, Princeton Univ. Press, 1971.

[11] J. Neukirch, Class Field Theory, Springer, Berlin, 1986.

[12] H. Qin, The 2-Sylow subgroups of $\mathrm{K}_{2} \mathrm{O}_{F}$ for real quadratic fields $F$, Science in China Ser. A 23 (12) (1993), 1254-1263 (in Chinese).

[13] - , The 2-Sylow subgroups of the tame kernel of imaginary quadratic fields, Acta Arith. 69 (1995), 153-169. 
[14] H. Qin, The 4-rank of $\mathrm{K}_{2} \mathrm{O}_{F}$ for real quadratic fields $F$, ibid. 72 (1995), 323-333.

[15] J. Tate, Relations between $K_{2}$ and Galois cohomology, Invent. Math. 36 (1976), 257-274.

[16] E. Weiss, Algebraic Number Theory, McGraw-Hill, 1963.

[17] Q. Yue and K. Feng, The 4-rank of the tame kernel versus the 4-rank of the narrow class group in quadratic number fields, Acta Arith. 96 (2000), 155-166.

Institute of Mathematics

Fudan University

Shanghai 200433, P.R. China
Department of Mathematics Xuzhou Normal University Jiangsu 221009, P.R. China

E-mail: yue-qin@263.net

Received on 13.10.1998

and in revised form on 29.6.2000 